Monatsschrift f. Geburtshülfe u. Gynäkologie 1928;80:I-IV

\title{
Contents, Vol. 80, 1928
}

Inhaltsverzeichnis.

Originalarbeiten. S $\quad$ ite

Albrecht, Hans, Ehelicbe Fruchtbarkeit und Geburtenrückgaag in

Europa 98

Beck, Walter, Erfahrungen rait Avertin 272

Benthin, W., Átiologie und Klinik der korporalen Blutungen in der

Menopause . 117

Con ill, Victor, Der Schmetterlingshandgriff nach Proubasta zur Er-

weiterung des Muttermundes 442

Datnow, M. M., Zu dem Problem der Beliandlung bösartiger Geschwülste 243 Dyroff, R., Das

histologische Heilungsbild des Karzinoms nach Röntgen-

bestrahlung 153

Füth, H., Weiterer Beitrag zur Schwangerschaftsübertragung nebst Be-

merkungen über den Zeitpunkt des ersten Auftretęs des Vomitus

matutinus 260

Gerdessen, Kurt, Schweigepflicht und Meldepflicht des Geburtshelfers 318 Henrard, E., Zur

Átiologie und Prognose des Genu recurvatum con-

genitum beim Neugeborenen 317

Hinselmann, Hans, Karzinomatöse Portioleukoplakie?

Herrmann, Albert, Granulosazellgesehwulst des Ovars 143

de Jongh, S. E., siehe Laqueur, Ernst.

Isbruch, Friedhelm, Zur Frage der metastatischen gastroenterogenen

Ovarialkarzinome (Krukenberg-Tumoren) 289

Kallinikoff, J., 1000 künstliche Schwangerschaftsunterbrechungen

(Abortus arte provocatus, Embryothrypsia) 109

v. Khreninger-Guggenberger,J., Blutgruppenuntersuchungen am

Retroplazentar- und Nabelschnurblut 104

Laqueur, Ernst, und S. E. de Jongh, Zur Wirkung des weiblichen

Sexualhormons Menformon, im besonderen auf die Mamma; zugleich

ein Beitrag zur Bedeutung der Dosierung von biologisch wirksamen

Präparaten 425

Lork, Erich C, Über puerperale Gangrän

Martin, Ed., Dammschutz und Dammschnitt 412

Mgalobeli, Michael, Beitrag zur Frage der fieberhaften Aborte . . 265 Naujoks, H, Das spätere Schicksal der Kinder mit intrakraniellen

Geburtsläsionen 297

Rosenloecher, Karl, Über Appendizitis und mensuellen Zyklus . . 403

Schmid, H. H., Haematokolpos lateralis 283

Schmitt, Walter, Über die spätere Entwicklung der Kinder vorbestrahlter 
Frauen 157

-Schneider, G. H, Die Ergebnisse der Milzbestrahlungen in Kombination

mit Röntgentherapie bei gynäkologischen Erkrankungen 146

Schubert, G., Bemerkungen zu Wiemers Auf'satz (Band 79, S. 51): Die

Operation des Prolapses und der Retroflectio uteri fixata nach

Schubert $\quad 140$

Schwenke, Karl, Unsere Erfahrungen mit der Kiellandzange . ... 82 Sieber, Fritz,

Uterusexstirpation wegen chronischer Gonorrhoe ... 19 Spiegler, R., siehe Wiechmann, Ernst.

Vogt, E., Über die Beziehungen der Geburtsliilfe und Gynäkologic zu

Augenleiden 1

Erfahrungen mit Pernocton beim geburtshilflichen Dämmerschlaf . . 79

Über die prophylaktische Thrombosierung von Varizen 422

++++++++++++++++++++++++++++++++++++++++++++++++++++++++
++++++++++++++++++++++++++++++++++++++ jy Inhaltsverzeichnis.

Seite

Weill, L., Eeitrag zur Entwicklungsmechanik (Naclitrag) 278

Wiechmann, Ernst, Asthma bronchiale und Sehwangerschaft (Be-

merkungen zu der gleiclmamigen Arbeit von li. Spiegler in Bd. 79,

S. 193 dieser Monatsschrift 410

Luigi Mangiagalli $\dagger 242$

Sammelbericht.

Die Frage der Narkose und Anästhesie. Kritiscb.es Sammelreferat von

Heinz Küstner-Leipzig 29

Berichte fiber ausländische Literatur.

Jaliresbericht über die spanische geburtshilfliche und gynäkologische

Literatur. Von Prof. Dr. Victor Conill-Barcelona 38

Bericht über die italienische Literatur. 1928. I. Sem. Von Priv.-Doz.

Dr. Scbastiano di Francesco-Mailand 443

Vereíns- und Literaturbeilage.

Original-Sitzungsberichte aus geburtshilflich-gynäkologischen Gesellschaften:

Gesellschaft für Geburtshilfe und Gynäkologie zu Berlin.

Sitzungen vom 27. IV., 11. V. und 8. VI. $1928 \quad 53$

Sitzung vom 22. VI. $1928 \quad 160$

Sitzung vom 13. VII. 1928335

Gynäkologische Gesellschaft zu Breslau. Sitzung vom 19. VI. 1928 . . 342

Münchener Gynäkologische Gesellschaft. Gemeinsame Sitzung mit der

Dermatologischen Gesellschaft am 16. II. $1928 \quad 60$

Südostdeutsche Gesellschaft für Geburtshilfe und Gynäkologie. IV. Ta-

gung am 25. und 26. II. 1928 in Breslau 64

Mittelrheinische Gesellschaft für Geburtshilfe und Gynäkologie.

Sitzung in Frankfurt a. M. am 26. II. 1928166

Sitzung in Frankfurt a. M. am 20. V. 1928352

Bayerische Gesellschaft für Geburtshilfe und Frauenheilkunde. Sitzung

am 11. XII. 1927 zu Nürnberg

179

Niederrheinisch-westfälische Gesellschaft für Gynäkologie und Geburts

hilfe. 77. bis 81. Sitzung 207 
82. bis 86 . Sitzung $\quad 367$

Nordwestdeutsche Gesellschaft für Gynäkologie. Sitzung vom 12. V. 1928348

Die geburtshilflich-gynäkologische Abtcilung auf der 90. Versammlung

der Gesellschaft deutscher Naturforscher und Ärzte in Hamburg am

16. bis 22. IX. 1928. Von E. Пöhne-Greifswald

391

$\Lambda$ rztekonferenz über die Bekämpfung der Frühsterblichkeit der Neu-

geborenen am 16. IX. 1928. Von E. Martin-Elberfeld 393

90. Versammlung der Gesellschaft Deutscher Naturforscher und Ärzte am 16. bis 21. IX. 1928

zu Hamburg. Abteilung für Geburtshilfe und Gynäkologie. Von Dr. G. H. Schneider-

Brandenburg a. d. H. . . 395

Vom II. internationalen Kongreß der Strahlenkunde in Stockholm vom

23.-28. VII. 1928. Von Dr. G. H. Schneider-Brandenburg . . 456

Geburtshilflich-gynäkologische Gesellschaft in Wien.,, Sitzungen vom

8. V. und 12. VI. 1928

162

Buchbesprechungen 237, 402

Sachregister für die Bände 76-80 461

Namenregister für die Bände 76-80 482 